\title{
Investigation of the Quality of Antibiotics-Based Amoxicillin for Monitoring of Some Different Medicine Markets of Democratic Republic of Congo
}

\author{
Nicodème Kalenda Tshilombo1,2, Patient Ciza Hamuli1,3, Jérémie K. Mbinze, \\ Védaste Habyalimana ${ }^{4}$, Dibungi T. Kalenda ${ }^{2}$, Don Jethro Mavungu ${ }^{5,6}$, Pierrot Mwamba ${ }^{7}$, \\ Philippe Hubert ${ }^{1}$, Roland Djang'eing'a Marini ${ }^{*}$
}

\author{
${ }^{1}$ Laboratory of Analytical Chemistry, CIRM, Department of Pharmacy, Liege University, Liège, Belgium \\ ${ }^{2}$ Laboratory of Chromatography, Faculty of Pharmaceutical Sciences, University of Kinshasa, Kinshasa, \\ Democratic Republic of Congo \\ ${ }^{3}$ Laboratory of Drug Analysis, Department of Galenic Pharmacy, University of Kinshasa, Kinshasa, Democratic Republic of Congo \\ ${ }^{4}$ Medical Procurement and Production Division, Rwanda Biomedical Center, Kigali, Rwanda \\ ${ }^{5}$ Laboratory of Pharmacognosy, CIRM, Department of Pharmacy, Liege University, Liège, Belgium \\ ${ }^{6}$ Ecole Régionale Postuniversitaire d'Aménagement et de Gestion Intégrée des Forêts et Territoires Tropicaux, University of \\ Kinshasa, Kinshasa, Democratic Republic of Congo \\ ${ }^{7}$ Department of Pharmacy Galenic, University of Lubumbashi, Lubumbashi, Democratic Republic of Congo \\ Email: *rmarini@uliege.be
}

\begin{abstract}
How to cite this paper: Tshilombo, N.K., Hamuli, P.C., Mbinze, J.K., Habyalimana, V., Kalenda, D.T., Mavungu, D.J., Mwamba, P., Hubert, P. and Marini, R.D. (2018) Investigation of the Quality of Antibiotics-Based Amoxicillin for Monitoring of Some Different Medicine Markets of Democratic Republic of Congo. American Journal of Analytical Chemistry, 9, 366-385. https://doi.org/10.4236/ajac.2018.98029
\end{abstract}

Received: March 16, 2018

Accepted: August 6, 2018

Published: August 9, 2018

Copyright (c) 2018 by authors and Scientific Research Publishing Inc. This work is licensed under the Creative Commons Attribution International License (CC BY 4.0).

http://creativecommons.org/licenses/by/4.0/ (c) (i) Open Access

\begin{abstract}
In order to combat the counterfeiting of drugs, adapted HPLC analytical USP methods were applied to evaluate the quality of the amoxicillin (with or without potassium clavulanate) powder for suspension sold in some Congolese markets. The adaptation has been done by modifying the column dimensions and adjusting the flow rate. According to the intended deployment of these methods in Democratic Republic of Congo (DRC), 3 factors (analyst, day and equipment) were involved in the validation step while applying the classic total error measurement approach with an accuracy profile as decision tool. Since adequate results were obtained in terms of selectivity, precision, trueness and accuracy (tolerance limits of life expectancy: $-6.0 \%$ and $3.8 \%$ ) for levels of interest concentration, the methods have been considered for routine use on several samples from different provenances and collected in 4 major DRC cities. Out of 278 samples collected, 200 were eligible for analysis from which $28 \%$ were found under standards with several figures: $\mathrm{pH}$ failure, out of specification for amoxicillin content, absence of potassium clavulanate, physical modifications of the powders. As evidenced by these findings, medicines
\end{abstract}


of low-quality continue to be a major public health problem requiring appropriate action to effectively address this problem.

\section{Keywords}

Poor Quality Medicines, Amoxicillin, LC Method, Validation, Survey Study

\section{Introduction}

The quality of medicines becomes more and more problematic and particularly regarding poor quality [1] [2] [3] [4] [5]. This problem is more worrying in lowand middle-income countries (LMICs) [6] [7]. There is insufficient information on the magnitude of this scourge and few global studies on this issue are available for Democratic Republic of Congo (DRC). However, the phenomenon affects developed countries in a certain extend and mostly developing countries where medicines manufacturing, import, supply and distribution activities are less regulated and even not at all, and where the application of Regulation is problematic and characterized by the lack or insufficiency of drug control, the random supply of drugs, corruption and interest. The consequences and dangers of poor quality medicines are multiple: therapeutic failure, drug poisoning, microbial resistance or even death [8]. Children are the most vulnerable [9]. According to P. Newton's definition, low quality drugs encompass falsified/counterfeit, substandard or degraded drugs [10]. This definition seems practical and adapted to the study in the present paper. However, a counterfeit or falsified medicine is not necessarily of inferior quality in terms of content. The counterfeit products may contain no active ingredient, another active ingredient, or the correct active ingredient but at an incorrect dosage. From the point of view of falsification, the problem of medicines of low quality is an economic crime that discredits the pharmaceutical industry and the threat of bankrupt. The impact of poor quality medicines is a real, worldwide and multifaceted threat to public health [11]. According to the World Health Organization (WHO), more than $30 \%$ of medicines are counterfeit in some areas of Latin America, South East Asia and Sub-Saharan Africa. In emerging economies countries, the proportion is estimated at $10 \%$ but in some of those countries for example the former Soviet republics it can be as high as $20 \%$. In wealthy countries, with strong regulatory mechanisms, counterfeits account for less than $1 \%$ of the market value, but $50 \%$ of illegal Internet sales are counterfeit. The world market medicines are of about $10 \%$ low quality in developing countries, and more than $30 \%$ in some regions of sub-Saharan Africa [12]. Nearly $28 \%$ of inferior drugs belong to anti-infective. However, the majority of poor-quality anti-infective are from India (54\%), followed by China (21\%). More than $50 \%$ belongs to beta-lactam antibiotics whose amoxicillin remains the most cited. The most affected dosage forms are those for oral route administration (77\%), in particular capsules, tablets, syrups and suspension 
powders, which probably offer more ease of forgery because they require little sophisticated equipments [13] [14].

Infectious diseases are the main cause of infant mortality, with up to $36 \%$ observed between 1990 and 2015 in DRC [15], in contrast with use of pediatric based antibiotic, which is the first-line in several treatment diseases. However, in a poor-quality antibiotics environment, there is high risk of antimicrobial resistance increase with infant morbidity and mortality consequences [16].

The efforts made during the last decade by the major pharmaceutical industries to fight against falsification are not negligible in particular in the distinguish of fake medicine from true using new analytical techniques such as spectroscopy, X-ray, identification by radiofrequency of labels as well as the use of barcodes, holograms of invisible engravings and nanotechnology, etc. [17] [18]. These technologies have advanced the detection of falsified innovative products, but do not ensure protecting the generic products. However, if governments adopt to have a unique centre of labelling and packaging, these technologies would be useful to detect fake in generic medicines. In any case, the quality control of the drugs at the level of the distribution points (pharmaceutical wholesaler, medical center and pharmacy) is necessary to evaluate the quality of the drugs consumed by the population and thus to apprehend the tracks of the solutions to fight against poor quality drugs [19] [20].

Several modern analytical techniques used for drug analysis have been reported including separative (liquid chromatography, capillary electrophoresis) and non-separative ones (Near infrared, UV-visible spectrophotometry). The analytical method assays of amoxicillin and its combination with potassium clavulanate are reported either in literature or in the pharmacopoeias. Liquid chromatography (LC) appeared to be the most appropriate analytical technique regarding to its advantage in terms of selectivity, precision and robustness of its application in routine analysis when dealing with several types of samples from several manufacturers.

However, many of the developed LC methods indicated for routine analysis required appropriate optimization process of parameters which can be of high influence during routine analysis in low income countries. Those parameters include: flow rate, column specifications and mobile phase (buffer $\mathrm{pH}$, buffer composition and concentration, organic modifier proportion). Therefore, the attempt is to adapt those method (i.e. USP method) by modifying some of those parameters (in example column characteristics and flow rate) meanwhile maintaining the efficiencies of that method;

The present study aimed to investigate on the quality of amoxicillin (alone or associated to potassium clavulanate) (see Figure 1) oral suspension powders collected on the Congolese market (DRC). The LC methods used are those from United States Pharmacopeia (USP-37edition) (the referential from which we based our method using the amoxicillin monograph) [21], adapted and validated for routine application [12]. The purpose of the methods developed in this work 

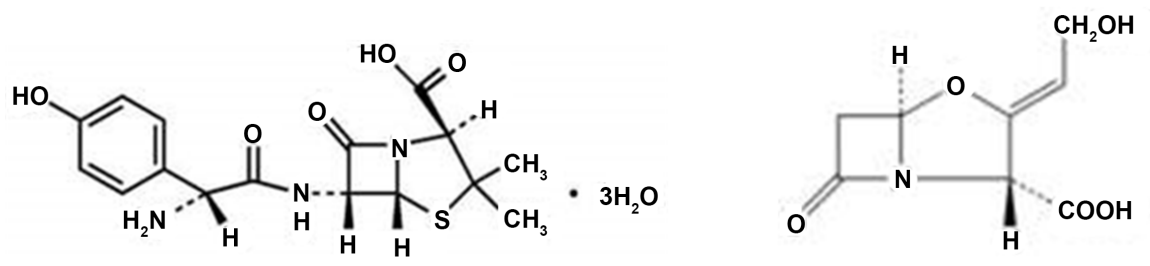

Figure 1. Chemical structures of amoxicillin trihydrate (left) and clavulanic acid (right).

being a long-term use in the DRC, the choice of the LC methods based on the USP and the resulting specifications were guided by the National Medicines Regulatory Authorities (NMRA) of DRC and some local manufacturers.

\section{Materials and Methods}

\subsection{Reagents and Chemicals}

Potassium hydroxide, potassium dihydrogen phosphate and orthophosphoric acid Eur Ph. grade (85\%) were purchased from Merck (Darmstadt, Germany). Acetonitrile HPLC far UV gradient grade was provided by Biosolve BV (Deventer, Netherlands). Methanol ultra-gradient HPLC was provided by J. T. Baker (Gliwice, Poland). Ultrapure water for mobile phases and preparation of samples was obtained from a Milli-Q Plus 185 water purification system from Millipore (Billerica, MA, USA). The reference substances of amoxicillin trihydrate (99.4\%) were purchased from Molekula GmbH (Germany) and of potassium clavulanate (99.8\%) from Sigma Aldrich (St. Louis, MO, USA). For the preparation of validation standards, matrix formulations were kindly provided by local manufacturers in DRC or obtained from classical literature for medicines imported from Europe and some from Asia.

\subsection{Instrumentations and Chromatographic Conditions}

The experiments of LC conditions including validation step and routine analyses were carried out on LC systems from Waters 2695 (Waters, Milford, USA; composed of a Waters selector 7678, autosampler and photodiode array detector PDA Waters 2996) and from Lachrom Hitachi (Kyoto, Japan); composed with classical LC modules including an autosampler, photodiode array detector PDA. The analytical column for preliminary experimentations was an XBridge C18 $(250 \times 4.6 \mathrm{~mm} ; 5 \mu \mathrm{m}$ particle size $)$ from Waters. Another XBridge C18 column $(100 \times 4.6 \mathrm{~mm}$ i.d.; $3.5 \mu \mathrm{m}$ particle size $)$ from Waters was used for validation and routine testing. Peak analytes were monitored by on-line UV detection considering the USP method namely at $230 \mathrm{~nm}$ for Amoxicillin only and at $220 \mathrm{~nm}$ for Amoxicillin associated with Potassium clavulanate. However, the UV spectra were on-line recorded from $200 \mathrm{~nm}$ to $400 \mathrm{~nm}$ to allow the peak identification at all the experiments. The injection volume was set at $10 \mu \mathrm{L}$ and the flow rate at $0.8 \mathrm{~mL} \cdot \mathrm{min}^{-1}$ while the column oven was maintained at $25^{\circ} \mathrm{C}$, with a run of 10 min for all tested experimental conditions. Measurements of $\mathrm{pH}$ were performed with a Seven Easy S20 pH-meter (Mettler Toledo, Columbus, OH, USA). 


\subsection{Software}

Empower 2.0 for Windows was used to control the Waters HPLC system and to record the signals from the detector for drawing the chromatograms and interpret them. In the same purpose, the software Manager was used to Lachrom Hitachi LC. The accuracy profiles as well as the statistical calculations including the validation results and uncertainty estimates were obtained using e-noval ${ }^{\circ} \mathrm{V} 3.0$ software (Arlenda, Belgium).

\subsection{Sample Preparation}

\subsubsection{Sample Solutions for Validation}

A stock solution of calibration standards (CS) of Amoxicillin (AM) $5.0 \mathrm{mg} \cdot \mathrm{mL}^{-1}$ was prepared in phosphate buffer at $50.0 \mathrm{mM}$ potassium dihydrogen phosphate adjusted to $\mathrm{pH}$ of $5.0 \pm 0.1$ with a $45 \%(\mathrm{w} / \mathrm{w})$ solution of potassium hydroxide. Several dilutions were performed in the same buffer in order to obtain running solutions at 3 different concentration levels to establish and evaluate the response function. In the same way, the stock solution of calibration standards with a concentration $500 \mu \mathrm{g} \cdot \mathrm{mL}^{-1}$ of amoxicillin and $62.5 \mu \mathrm{g} \cdot \mathrm{mL}^{-1}$ of potassium clavulanate (CL) were prepared in phosphate buffer at $50.0 \mathrm{mM}$ sodium hydrogen phosphate and adjusted to $\mathrm{pH}$ of $4.4 \pm 0.1$ with solution of potassium hydroxide at $45 \%(\mathrm{w} / \mathrm{w})$.

For the validation standards (VS), the solutions were prepared as for calibration standard including the reconstituted matrix powder for amoxicillin suspension and for mix of amoxicillin and potassium clavulanate. The stock solutions were obtained as in the case of calibration standards to which was added a corresponding amount of the matrix. Dilutions were performed in phosphate buffer in the same way as described for the CS in order to obtain five solutions at different concentration levels as shown in Table 1.

The solutions were freshly prepared and protected from light. After centrifugation at $5000 \mathrm{rpm}$ for 5 minutes, they were filtered through $0.45 \mu \mathrm{m}$ PTFE syringe filter discs before being placed into autosampler set in the LC analysis systems. Finally, 3 set of solutions were run and used for calibration standard and of 5 sets for validation standard considering that the $100 \%$ concentration level is the nominal level of the amoxicillin and of potassium clavulanate corresponding to the claimed values of samples.

\subsubsection{Sample Solutions for Routine Analyses}

After their validation, the methods have been implemented in a quality system environment to the use in routine analysis. This environment is under a European Union Good Manufacturing Practices (UE-GMP) licence authorisation $\left(\mathrm{N}^{\circ} 1813 \mathrm{H}\right)$ of the Pharmacy Department that is also WHO prequalified laboratory. The whole analyses were carried out according to an analytical procedure as shown in Figure 2.

The main tests were the $\mathrm{pH}$ measurements and the assay of active substances after their identification by two orthogonal tests namely comparison of 
Table 1. Matrix composition of several manufacturers.

\begin{tabular}{|c|c|c|c|c|c|c|c|c|c|}
\hline \multirow[t]{3}{*}{ Types of excipients } & \multirow[t]{3}{*}{ Excipients } & \multicolumn{8}{|c|}{ Manufacturers of powder for pediatric suspension } \\
\hline & & \multicolumn{6}{|c|}{$\mathrm{AM}$} & \multicolumn{2}{|c|}{$\mathrm{AM}+\mathrm{CL}$} \\
\hline & & LM1 & LM2 & LM3 & LM4 & EU1 & EU2 & AS & UE \\
\hline \multirow[t]{3}{*}{ Sweeteners: } & Carmelose $\mathrm{Na}$ & - & - & - & - & + & + & - & + \\
\hline & Aspartame & - & - & - & - & + & + & - & + \\
\hline & Saccharin $\mathrm{Na}$ & + & + & + & + & - & - & - & - \\
\hline \multirow[t]{5}{*}{ Thickener: } & Xanthan gum & - & - & - & - & + & + & - & + \\
\hline & $\begin{array}{l}\text { Anhydrous colloidal } \\
\text { silica }\end{array}$ & - & - & - & - & + & + & + & + \\
\hline & $\mathrm{Na}-\mathrm{CMC}$ & + & + & + & + & - & - & + & - \\
\hline & Crospovidone & - & - & - & - & + & + & - & + \\
\hline & Simeticone & - & - & - & - & - & - & + & - \\
\hline Humectant: & Mg Stearate & - & - & - & - & + & + & + & + \\
\hline Preservative: & Na Benzoate & + & + & + & + & + & + & + & + \\
\hline Flavor + dye: & & + & + & + & + & + & + & + & + \\
\hline
\end{tabular}

Legend: (+): presence of the substance mentioned on the left; (-): absence of the substance mentioned on the left. (LM): local manufacturer; (EU): imported from Europe; (AS): imported from Asia; (AM): Amoxicillin; (CL): Potassium Clavulanate.
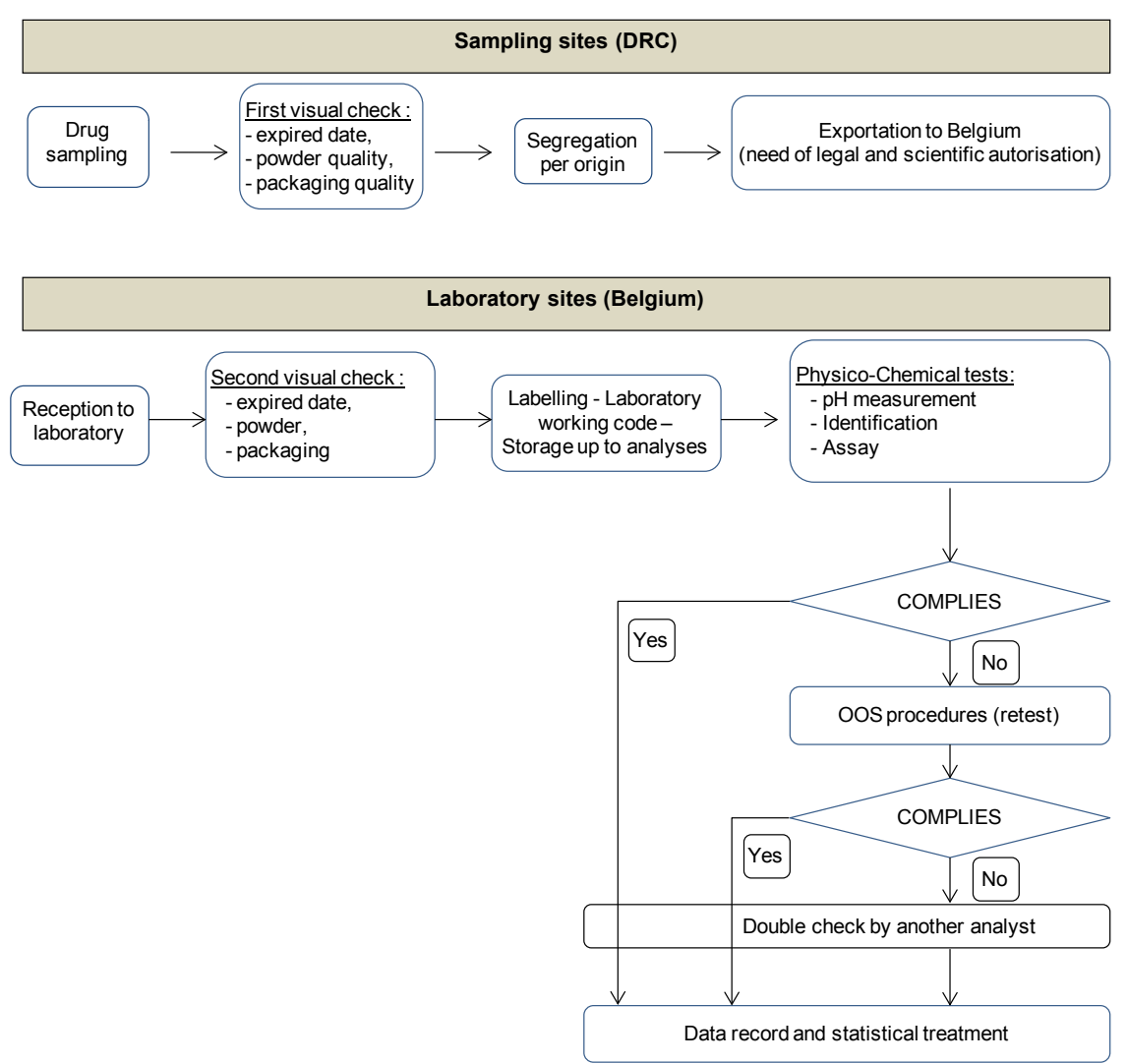

Figure 2. Flowchart of the whole analysis procedure from sampling to data treatment. 
the peak retention time with that of the reference substance and superposition of the respective UV-Vis spectra. The assay specifications were those of USP 37 that are $90.0 \%-120.0 \%$ for amoxicillin; $90.0 \%-125.0 \%$ for potassium clavulanate and $5.0-7.5$ for $\mathrm{pH}$ of the reconstituted suspension [12]. The nonconforming samples were reanalyzed by another analyst before concluding on the result.

\subsection{Sampling}

\section{Sampling Sites, Criteria and Sampling Size}

Drug sampling for routine analyses was randomly done at Kinshasa, Matadi, Lubumbashi and Kolwezi cities (see sampling points at Figure 3).

These DRC cities are the main entrance of trade market as well for pharmaceutics and have intensive commercial activities (including pharmaceutics). In addition, they have large population. Kinshasa the political and administrative capital where the majority of official pharmaceutical distributors are based, has also the largest airport and one of the most active rive port on the continent connecting with Brazzaville in Republic of Congo, where there are distributors of Official French medical products. Lubumbashi is the second largest city in the country, after Kinshasa, in terms of population and economic traffic. Matadi, a city in western DRC like Kinshasa, is home to the biggest sea port in the country, allowing high traffic of goods from all over the world, mainly from Asia and Europe. Located in the south of the country, Kolwezi is a city close to Lubumbashi, but which is not bordering.

Sample size $(N)$ was estimated using the following formula [22]:

$$
N=p(1-p) z^{2} / E^{2}
$$

with $p$ the prevalence, $z$ the confidence interval and $E$ the margin error.

Since for antibiotics in DRC the exact prevalence is not available, we assumed a prevalence of $30 \%$ ( $p=0.3$ ) for poor quality medicines in some regions of sub-Saharan Africa [12]. With the margin of error $(E)$ of $10 \%$ and $95 \%$ of confidence interval $(z=1.96)$, the random sample will be estimated to 81 samples.

\section{Results and Discussion}

\subsection{Method Selection and Adaption}

This study was carried on the frame of methods transfer from Belgium to DRC, based on a specific lifecycle method. As the first step of that lifecycle, the method selection was done. We based our method orientation to the USP monographs with reversed phase LC using UV detection since several manufacturers or importers in DRC are claiming to follow that referential. Two selected USP methods were adapted in order to obtain short analysis time and reduced costs while maintaining their efficiency (see supplementary data). The adaptation allowed calculating the reduce of the mobile phase consumption to 6 folds while reducing the analysis time at 2 -folds. The latter is interesting to avoid the degradation of 


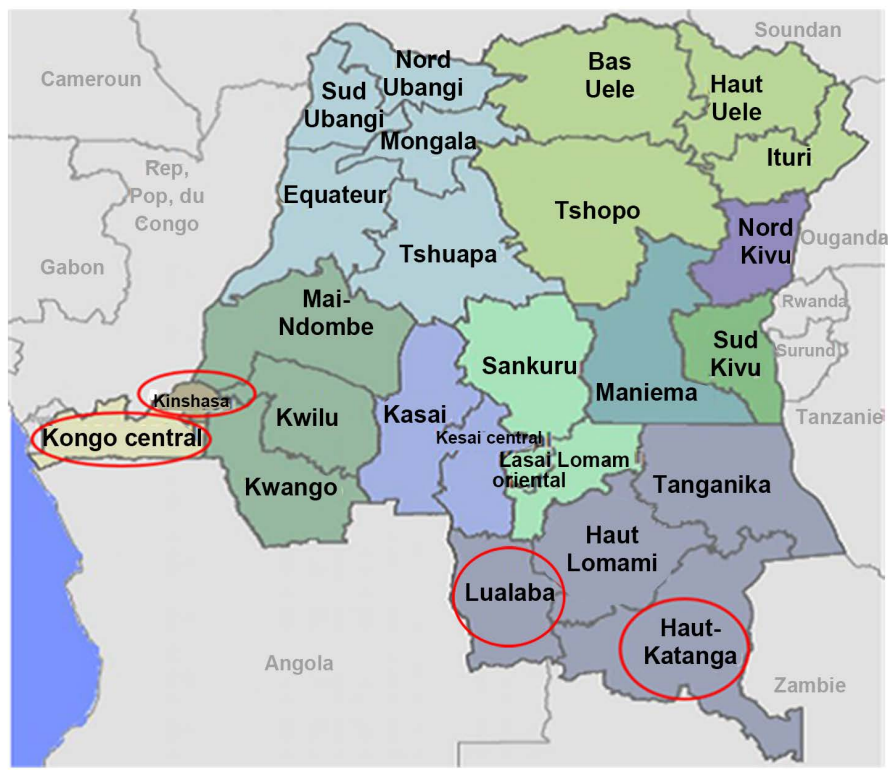

Figure 3. Map of the DRC; The red circles indicate the sampling sites: Kinshasa, Kongo central (at Matadi city), Haut Katanga (at Lubumbashi city) and Lualaba (at Kolwezi city). Source: https://www.investindrc.cd/en/drcongo-at-a-glance/drc-maps, (Copied on July 06, 2017).

active ingredients samples during their analysis. Remind that amoxicillin and potassium clavulanate degrade in aqueous media within 6 hours and 1 hour, respectively. Another objective was to use a standard method accepted by authorities, local quality control laboratories and even local medicines manufacturers/importers. Notice the USP based methods have to be appropriate for several conventional matrix types found in the market.

\subsection{Experimental Design for Validation}

At first, the selectivity of the two methods (Meth 1 and Meth 2) was evaluated by checking any possible matrix effect. The determination of matrix effect should provide information about any interference of the quantitative analysis. For that purpose, we analyzed the matrix solution of the most common and available matrices (Table 2).

Considering the chromatograms obtained for the most used excipients, no matrix effect was observed since there was no interference of the peak due to sodium benzoate with a resolution of 3 between its peak and that of amoxicillin in both methods (Figure 4).

In the next step of validation process, we applied the classic total error strategy with accuracy profile as a decision tool [23]. This strategy, which takes into account the validation parameters recommended by ICH Q2(R1), associates the dosage interval while estimating the probability of having an error based on the objectives of the method. To obtain the data necessary to estimate these validation parameters, including trueness, fidelity and bias, we performed an 
Table 2. Concentration levels (in $\%$ and in $\mu \mathrm{g} \cdot \mathrm{mL}^{-1}$ ) for validation for amoxicillin alone and for amoxicilline associated with potassium clavulanate.

\begin{tabular}{cccccc}
\hline \multirow{2}{*}{$\begin{array}{c}\text { Levels and the } \\
\text { correspondence (in \%) }\end{array}$} & \multicolumn{2}{c}{$\mathrm{AM}\left(\mathrm{in} \mu \mathrm{g} \cdot \mathrm{mL}^{-1}\right)$} & \multicolumn{2}{c}{$\mathrm{AM}\left(\mathrm{in} \mu \mathrm{g} \cdot \mathrm{mL}^{-1}\right)+\mathrm{CL}\left(\mathrm{in} \mu \mathrm{g} \cdot \mathrm{mL}^{-1}\right)$} \\
\cline { 3 - 6 } & $50 \%$ & Calibration Standard & Validation Standard & Calibration Standard & Validation Standard \\
\hline 2 & $75 \%$ & -50 & 75 & $50+6.25$ & $50+6.25$ \\
3 & $100 \%$ & 100 & 100 & $100+12.50$ & $75+9.38$ \\
4 & $125 \%$ & - & 125 & - & $100+12.50$ \\
5 & $150 \%$ & 150 & 150 & $150+18.75$ & $125+15.63$ \\
\hline
\end{tabular}
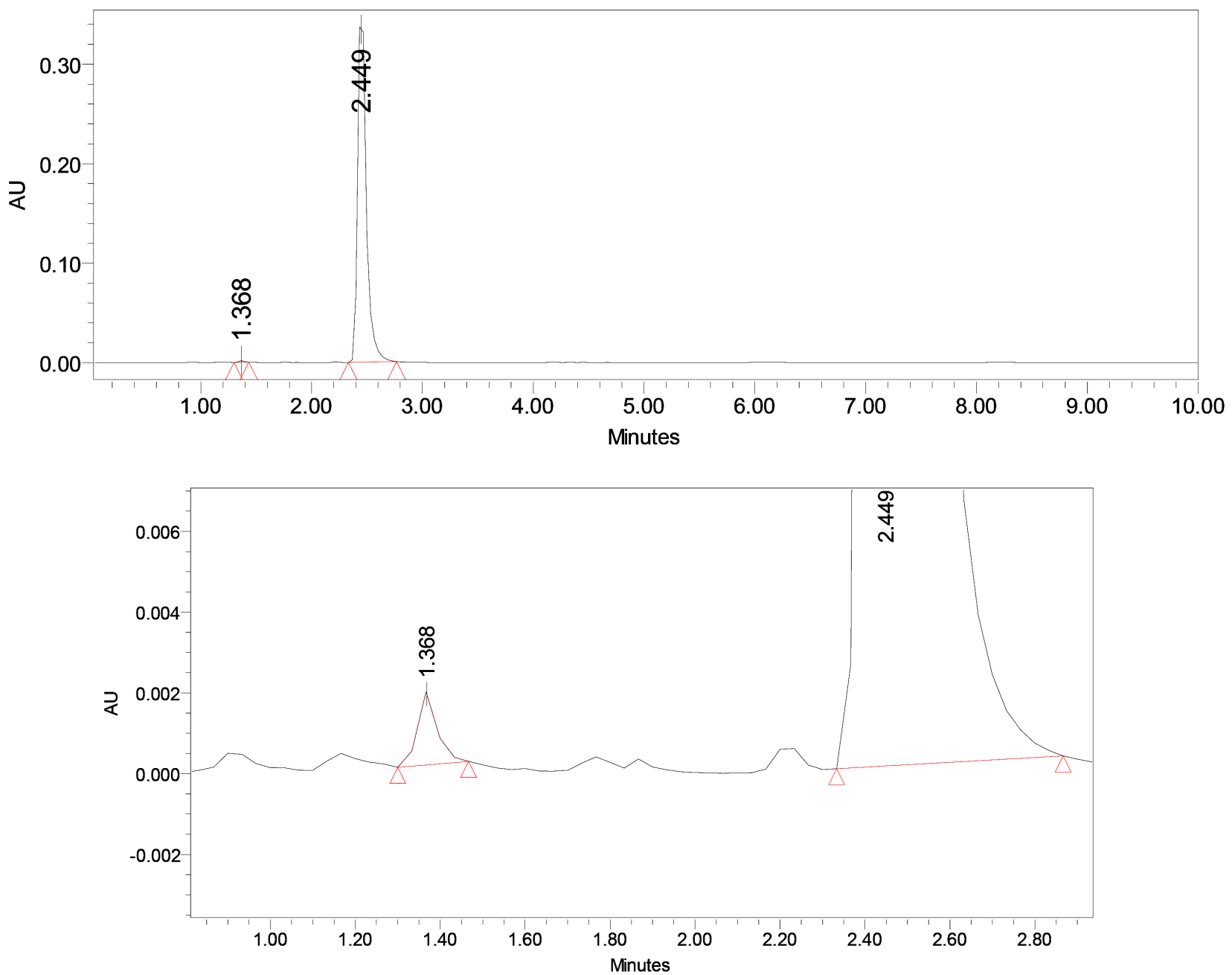

Figure 4. Illustration of a chromatogram of the Meth 1 where the concentration of sodium benzoate (retention time at 1.368 min) was intentionally increased to demonstrate the separation of its peak from that of amoxicillin (retention time at $2.449 \mathrm{~min}$ ).

experimental plan of 3 levels $(50 \%, 100 \%$ and $150 \%)$ for calibration standards and 5 concentration levels $(50 \%, 75 \%, 100 \%, 125 \%$ and 150\%) for validation standards. For each concentration level four replications were run for 4 days corresponding to 4 series $(p=4)$ to reduce the impact of the random error. 
The experimental plan of the validation took into account several operational factors namely the operator, the day and the equipment (see supplementary data). A series represents test results obtained under identical operating conditions (same operator, equipment and standards) in a given time interval (day). The standard deviation of these results, called repeatability, makes it possible to estimate the performance of the analytical system [24]. Intermediate fidelity (the inter-serial deviation) under conditions as representative as possible of the variability of the use makes it possible to estimate the performance of the analytical system routinely [25]. By combining 2 different operators and 2 equipment of different manufacturers we obtained 4 possible independent combinations for each series in order to detect and correct a possible systematic error. Generally, a validation plan of an analytical method considers a minimum of 3 days of performance testing that is statistically sufficient to estimate the performance of the method for its application in routine. However, this will consider only the effect due to time. Since we are expecting the method be used with several equipment and different analysts, we included the effects due to those two factors by testing different equipments and different analysts. Thus this will allow demonstrating the method performance including Intermediate Precision time-operator-equipment different.

First of all, the fixation of acceptance limits was discussed. Normally one considers the interval between the lower or upper content level around to the target value. However, based on the USP which levels are not symmetric $(90.0 \%$ to $120.0 \%$ ) for amoxicillin against the nominal content, the acceptance limits should be asymmetrical of $-10 \%$ to $+20 \%$. However, for easier of use, we set to \pm $10 \%$ that also is stricter for the upper limit. The same $\pm 10 \%$ acceptance limits were set up for potassium clavulanate which levels are asymmetrical also $(90.0 \%$ to $125.0 \%)$.

Then, we used the data (API concentrations and corresponding peak areas) of the calibration standard to establish several regression models that were tested against the validation standards to obtain the back-calculated concentrations of AM alone, AM and CLA in mixture. The accuracy profiles (see Figure 5) were established using the calibration curve of a linear regression through 0 and level $100 \%$.

It can be seen that the beta expectation tolerance intervals are included within the $10 \%$ acceptance limits expressed by the relative errors for both active ingredients and in the two methods. For amoxicillin, we have noticed a negative bias out of the acceptance limits at the concentrations above $140 \%$. The summary of the validation criteria tested is presented in Table 3.

It can be seen very good results for trueness with the highest value of relative bias below $2.3 \%$ at high amoxicillin concentration levels, for precision with the highest RSD value (2.8\%) observed only at the level of amoxicillin and for accuracy. The linearity and the different LOQ values were also acceptable to allow a reliable quantification in routine analyses. 


\section{Amoxicillin}

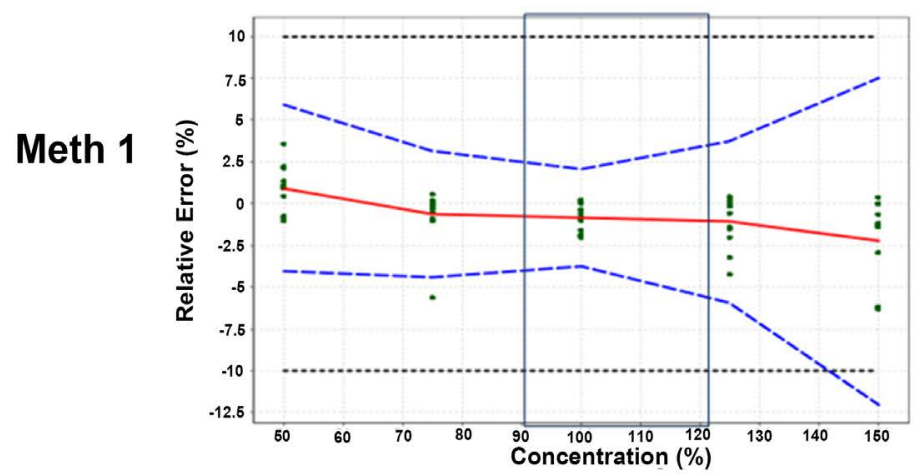

Amoxicillin

\section{Potassium clavulanate}

Meth 2
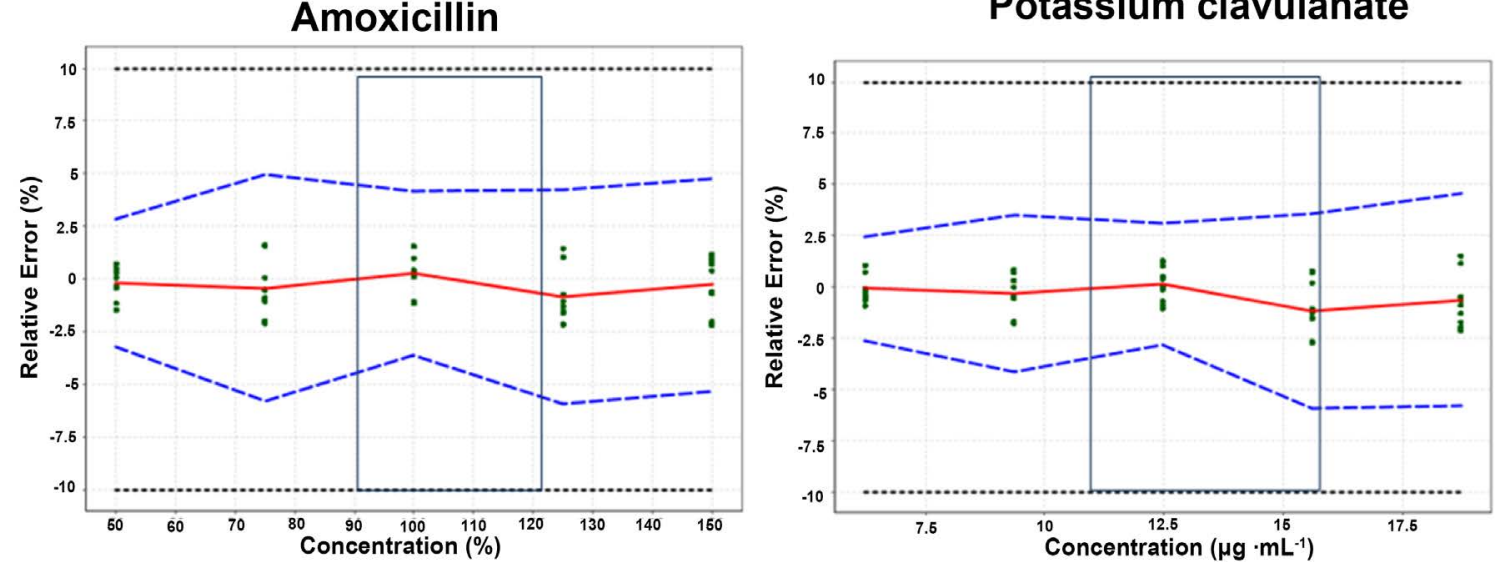

Figure 5. Accuracy profiles for quantitative Meth 1 (amoxicillin alone) and Meth 2 (amoxicillin and potassium clavulanate). $\underline{L e-}$ gends: Continuous red lines are relative bias; Dotted lines (in black) are acceptance limits ( $\pm 10 \%)$; Dashed lines (in blue) are $95 \%$ $\beta$-expectation tolerance intervals; Dots (in green) are relative biases at different concentration level measurements.

\subsection{Routine Applications}

\subsubsection{Consideration in sampling}

The sampling strategy for routine analysis was inspired from P. Newton proposal [26].

We would prefer large size but due to financial limitation, however we collected 278 samples. Samples were bought by anonymous shoppers as patients in random sampling method. Each collector behaved as in real shopping situation called technique of "blind procedure". This sample size seems enough for a pilot and preliminary study to assess the quality of pediatric formulations. Purchasers who were unaware of the study were recruited from the Kinshasa School of Public Health, from the Faculties of Pharmacy of Lubumbashi and of Kinshasa and the Medical Institutes of Kolwezi and of Matadi. Purchases were done in Circuit Urban Controlled (CUC) and Peri Urban Circuit Deficiency Controlled (PCDC) between November 2014 and November 2015. We were able to collect a total of 278 samples, 120 from CUC and 158 from PCDC. Then a first visual inspection based on the identification of the product, its validity (expired date) and the integrity of its packaging was carried out in Kinshasa by pharmacist's collaborators. A first encoding of the samples was done to identify samples as per their 
Table 3. Summary of the validation criteria for powder for suspension of AM (Meth 1) and powder of suspension for AM + CL (Meth 2).

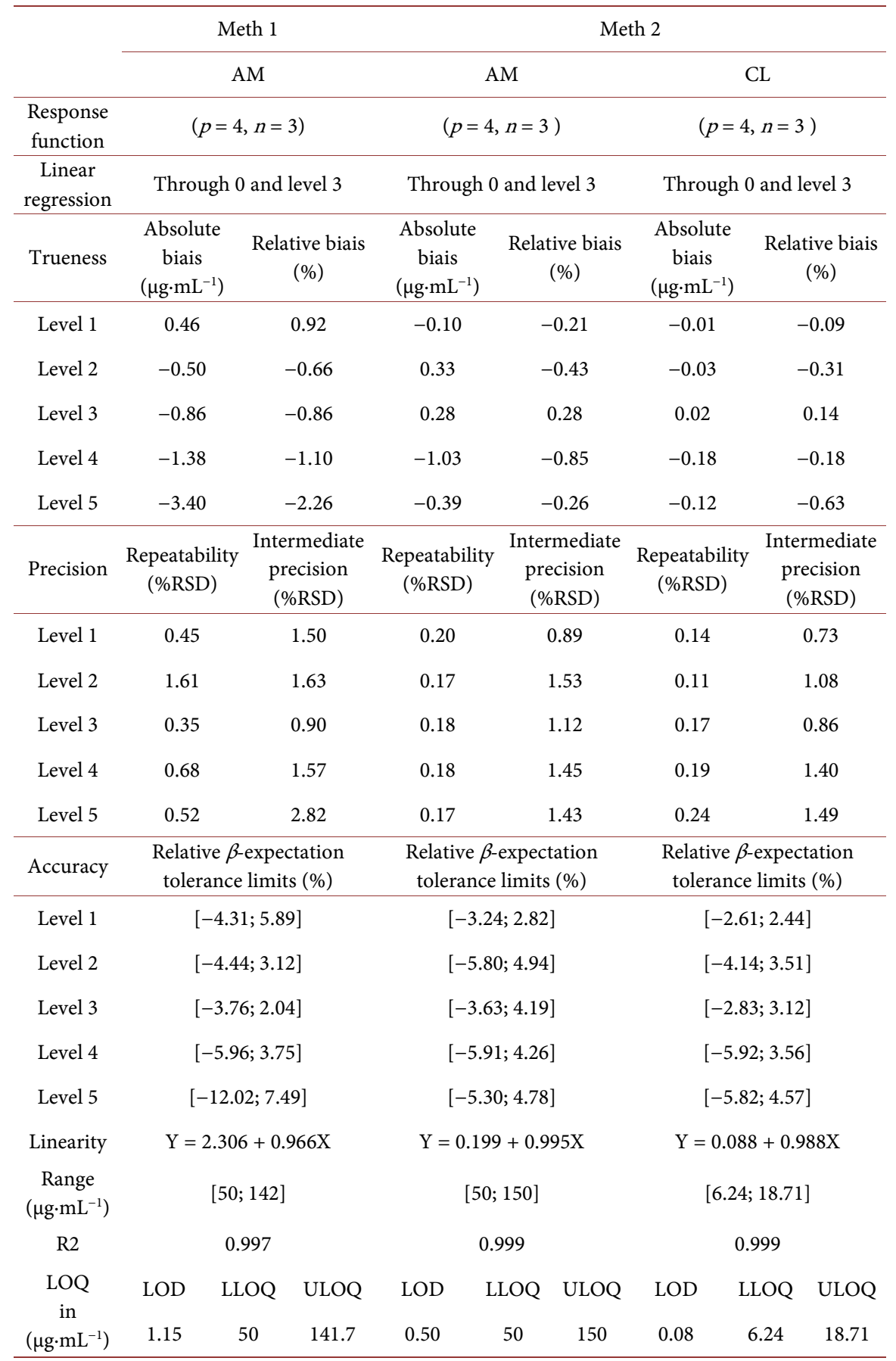

Legend: $\mathrm{p}=$ number of series; $\mathrm{n}=$ number of repetitions; LOD: limit of detection; LLOQ: Lower Limit of quantitation; ULOQ: Upper Limit of quantitation.

origin (manufacturer), place of purchase, expiry and manufacturing date, lot number, international non-proprietary name, brand name and the reported or claimed unit dose. Only 92 from CUC and 108 from PCDC were able to be analyzed in Belgium, the remaining were withdrawn due to not fulfilling our requirements above. Indeed, there were samples with not satisfying appropriate 
expired date (exceeded or less than one year expired date), the powder aspect that was clogged, the primary and secondary packaging that was not readable, some were even absent. In addition, insufficiency of unit number per sample was applied to discard not valid samples. To prevent retest analysis, the eligible samples should have at least two units. Than samples were stored in controlled environmental conditions (see section 2.5.1.). From the total eligible samples (200 samples), $48.0 \%$ samples were manufactured in the DRC, 30.5\% in France and $21.5 \%$ in India.

Depending on the sampling place and thus their storage conditions by located population, the samples were categorized into two circuits: The Circuit Urban Controlled (CUC) and the Peri Urban Circuit Deficiency Controlled (PCDC). The controlled circuit is the one which is under the control of the Pharmacy Direction of the Ministry of Health (Direction de la Pharmacie et du Médicament or DPM) that is the National Medicines Regulatory Authorities (NMRA) in the DRC. Apart the verification of authorization administrative documents, their duties are the control of the conservation conditions of medicines since DRC belongs to the climatic zone IVa $\left(\max 30^{\circ} \mathrm{C}, \max 65 \% \mathrm{RH}\right)$ [27]. The CUC samples were purchased from authorized wholesalers. These provide medical products at all levels in the public, private and denominational sectors in urban, peri-urban and rural areas. In addition, some of them are exclusive importers and distributors of products for the entire country. Normally, in this circuit the risk of finding falsified and degraded medicines is low. The PCDC is a circuit where control is almost not existing with high risk of encountering falsified and degraded products [28].

The samples were powder for pediatric suspension of Amoxicillin (AM) or associate with clavulanate of potassium $\mathrm{CL}$ ). The selection criteria mostly applied was the importance of drug in terms of distribution volume and therapeutic aspect; the active pharmaceutical ingredients have to be widely used in the DRC in the most recurrent infections in children according to the statistics of the National Health Development Plan 2010 [29]. Moreover, infant mortality in the DRC remains of concern [30] [31]. AM and CL fulfilled to these criteria. Other inclusion criteria mainly on technical aspects were valid expiring date, not clogged powder and intact primary packaging.

\subsubsection{Consideration in Analysis through Internal SOPs}

When received in Belgium, the samples were checked again to confirm they initial conditions and if they are still correct for analysis. An identification code and a bar code were assigned to the eligible samples. This code allowed administrative and physical traceability of the sample from receipt to the results issuing. Administrative traceability was supported by the following documents: request for analysis, receipt, raw results of the analysis, certificate of analysis, copy of the laboratory booklet and all associated records or documents. Identification and assay of active ingredients were done $\mathrm{pH}$ measurements as well.

For the analysis of amoxicillin samples, we used method 1 in which the con- 
centrations of standards solutions were $100 \mu \mathrm{g} \cdot \mathrm{mL}^{-1}$ diluted from a stock solution of amoxicillin at $1.0 \mathrm{mg} \cdot \mathrm{mL}^{-1}$ (100 $\mathrm{mg}$ of amoxicillin up to $100 \mathrm{~mL}$ ).

For samples containing both amoxicillin and potassium clavulanate, we used method 2 in which standards solutions contained also $100 \mu \mathrm{g} \cdot \mathrm{mL}^{-1}$ of amoxicillin but with $12.5 \mu \mathrm{g} \cdot \mathrm{mL}^{-1}$ of potassium clavulanate that were prepared from 100 $\mathrm{mg}$ of amoxicillin and $12.5 \mathrm{mg}$ of clavulanate up to $100 \mathrm{~mL}$.

In the two situations, two independent standard solutions were prepared. Since in solution, amoxicillin and potassium clavulanate are not stable especially at room temperature, we took care to perform the run of the set within less than 6 hours. In addition, the set was accepted if the recovery of the second standard compared to the first is between $98.0 \%-102.0 \%$.

Samples which are powder to be reconstituted with water were freshly prepared following the manufacturer's instructions on the package leaflet.

The overall results indicated that $28 \%(\mathrm{CI} ; 21.3 \%$ - 33.7\%) samples were declared out of specification (OOS). This finding is very close to the overall prevalence (30\%) of low-quality drugs in sub-Saharan countries as estimated by WHO [12]. In addition, a recent review of 44 prevalence studies in 25 different countries, including 11 low-income countries, shows that the median prevalence of substandard/counterfeit drugs was $28.5 \%$ (range $11 \%$ - 48\%) [32]. On the other hand, this gross result seems to be lower than the estimates of the review of the literature on the global prevalence (more than 50\%) of antimicrobial directed in 2015 by T. Kelesidis [14].

From the detailed results we noticed 7 samples (or 3.5\%, CI: $0.9 \%-6.1 \%$ ) with $\mathrm{pH}$ values not complying, of which 3 cases were the sole cause of the non-specification of the sample. Also 52 samples were OOS because of AM and/or CL content, 4 cases of overdosage, 2 cases of absence of potassium clavulanate, one case with a $\mathrm{pH}$ greater than 7.5.

Table 4 shows the OOS results divided into two circuits, the CUC that is recognized by DPM and the PCDC with uncertain or deficient control. There was non-significant difference in quality between locally manufactured medicines and those of European manufacturers in the CUC.

According to the result and considering the GMP of European manufacturers, the case of OOS noticed in certain European products may be due to the falsification of which these products are often victims since there is no traceability of original source. They might have a very probable hypothesis with the existence of mafia drug trafficking networks especially between Kinshasa and Brazzaville. The heads of African states, especially those of the two Congo, expressed their concern at the France Africa summit held in January 2017 in Bamako (Mali) [33]. In the light of these results, the frequencies of the poor-quality medicines in the periurban zone represented by the PCDC is much higher than those of the CUC. The prevalence of non-specification products is close to that observed by T. Kelesidis (see above). Of the 200 samples of powders for suspension based of amoxicillin a $46 / 55$ (83.6\%, CI: 73.9; 93.4) of the OOS samples were from PCDC. This indicates that the application of an effective quality control system and a 
Table 4. Number and percentage (\%) of samples out of specification the USP acceptance criteria. For each circuit (CUC and PCDC) by origin, AM associate or not and sampling site.

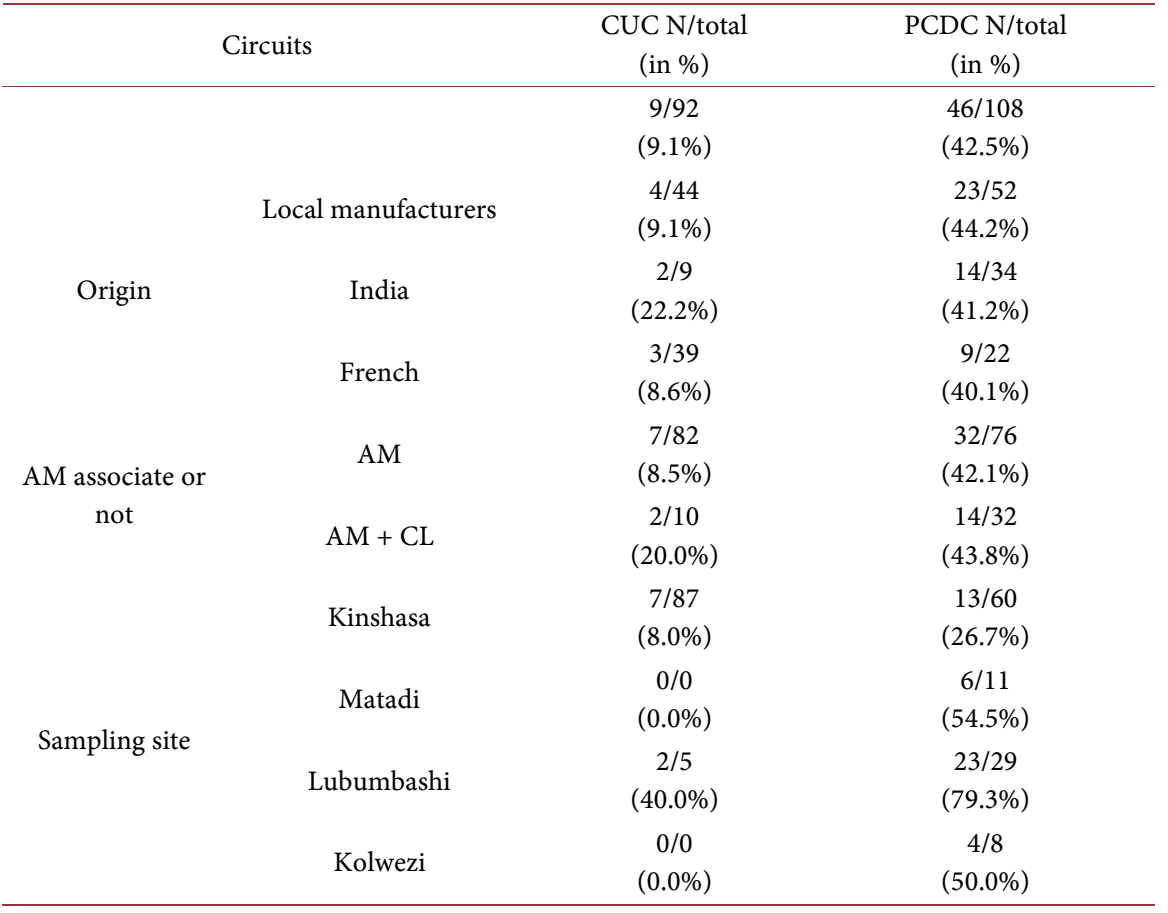

strong political commitment by the NMRA may reduce the prevalence of counterfeits [34]. With regard to the different origin the frequency of the drugs of bad is almost five times higher in the PCDC for the locally manufactured products (LM) and Indian origin (AS). This can be due to non-compliance with GMP rules during the manufacturing process or a counterfeit. Also, for the LM to the problem of packaging (non-hermetic primary packaging) and the conservation conditions are still not respected; Almost $10 \%$ of the samples were not analyzed because of the presence of visible traces of the powders on the primary packaging or because of the bulk of the powder. We can also observe that further away from Kinshasa (the central distribution point) the prevalence increases. It was in Kolwezi, the least accessible site of all, that there were two cases of CL. absence in two samples manufactured in India, whose official distributor based in Kinshasa did not recognize the batch. This reflects the lack of traceability of pharmaceutical products in DRC even among official distributors. (Figure 6 illustrates the revelation of the absence of clavulanate in a sample).

Apart from two cases due to non-compliant $\mathrm{pH}$, the overall assay results were presented according to their circuits the sampling site and the manufacturers (see supplementary data). We could notice that the distribution of OOS was dominated mainly by under dosage content than higher. However, the results within specification were somewhat in high proportion. Figure 7 confirms that distribution in particular for amoxicillin content for samples from peri-urban areas (PCDCs) that tends towards low values (under dosage).

The distribution of the OOS samples according to their origins in the different 

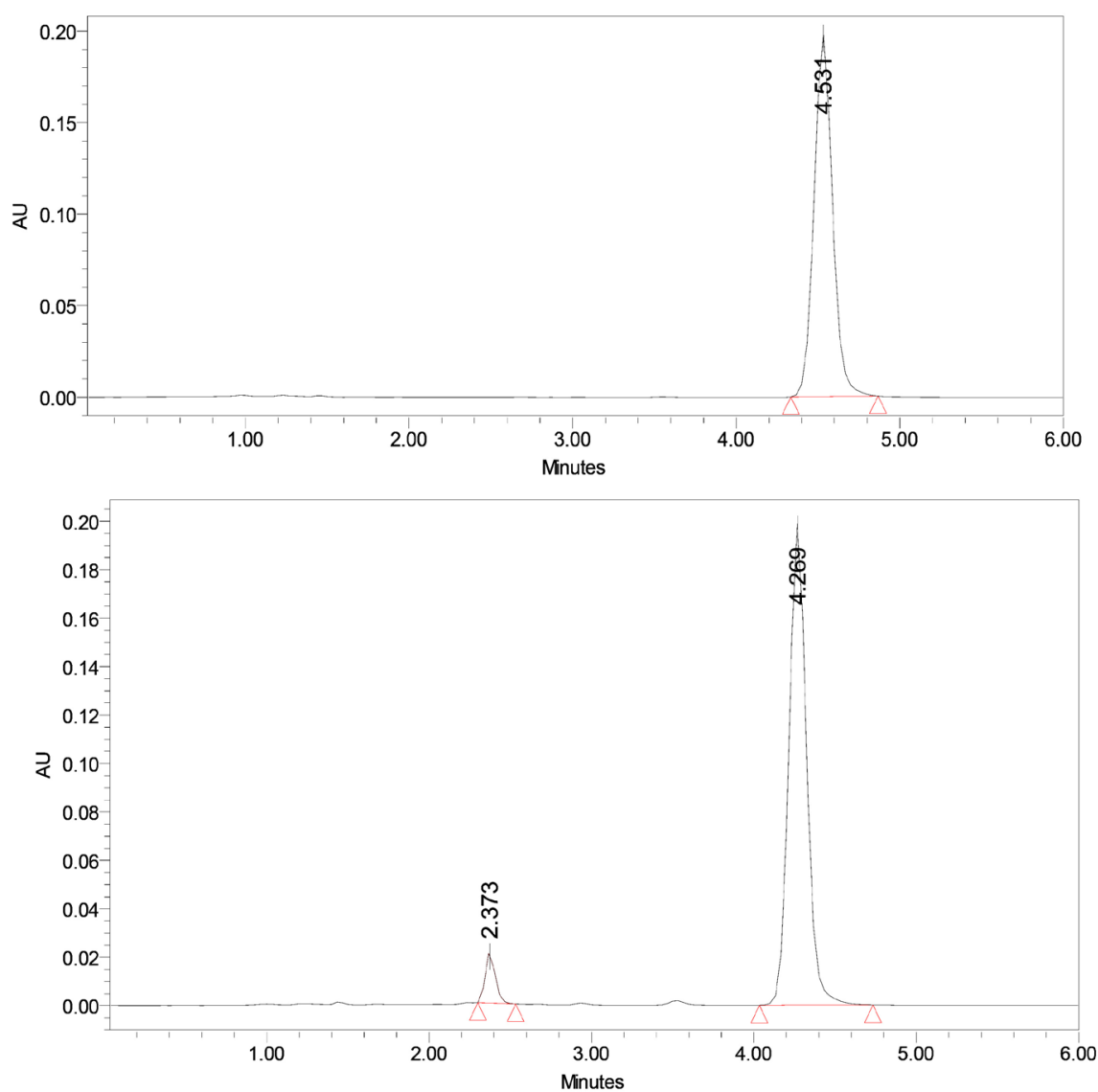

Figure 6. Illustration of a chromatogram of a powder sample for AM suspension claimed to contain $\mathrm{CL}$ which peak is absent. Above, on the left, the chromatogram of the substances of reference $(\mathrm{CL}+\mathrm{AM})$.
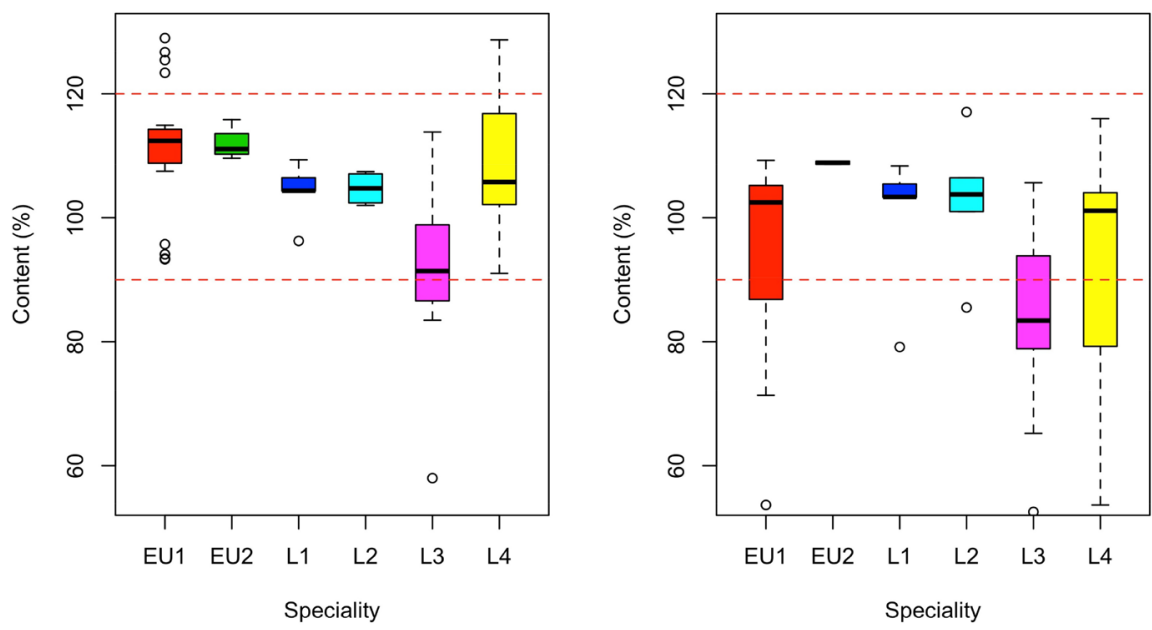

Figure 7. Trend of OOS results of AM in the CUC (right) and in the PCDC (left); EU1: European manufacturers (France), EU2: Asian manufacturers (India) L; Local manufacturers (DRC).

circuits reveals that the local manufacturers L2 and L3 showed much dispersed results in the two circuits (CUC and PCDC). A failure to comply with GMP 
rules would be a probable cause of poor quality. However, the trend of these results to the lower median values in the PCDC indicates a probable degradation of these products because in this circuit the storage conditions are not always favorable. Reversing the trend of the local manufacturer (L3) when moving from the CUC to the PCDC seems to show that the trend of the amoxicillin content to the low values does not distribute same way. If one accepts that the poor conservation conditions would be a significant factor of the low content of amoxicillin in the PCDC then it does not affect all products in the same way. For example the sealing of the bottle closure system could be one of the reasons. Indeed, the visual inspection carried out on these samples led to the discarding of 10 samples collected in the CPDC, 8 of which were from the local manufacturer L3 and 2 from the local manufacturer L2. These samples showed many lump, a sign of exposure to moisture. Interpretation for European manufacturer EU1, however it may suggest a counterfeit for the medicines of European manufacturers whose local representatives did not recognize the existence of certain batches (4 in total). It should be noted that information on the traceability of batches was not always easy to obtain either at the Ministry of Health or at the local manufacturers or the representatives of European manufacturers.

\section{Conclusions}

The prevalence of $28 \%$ in our study confirms the hypothesis that in the DRC the non-compliance with good manufacturing practices, poor storage conditions and fraud could be the causes of substandard drugs. Several cases of poor quality powder for oral suspensions of amoxicillin alone or in combination with clavulanate have been discovered. Our results show that this prevalence increases sharply (83.6\%) with the remoteness of the distribution center, especially in peri-urban areas where the lack of quality control in the DRC is evident. This demonstrates that inadequate quality control is a factor that aggravates the prevalence of poor quality drugs.

The methods used in this study, adapted and validated USP methods using a reconstituted matrix inspired by local manufacturers, demonstrated acceptable performance accuracy (tolerance limits of life expectancy: $-6.0 \%$ and 3.8\%). These are intended for routine application in the DRC after an analytical transfer and could contribute to the improvement of quality control in this country.

Our study initially targeted a study encompassing Kinshasa the economic capital (distribution and control center) and five other major cities in the provinces of the country respectively east (Goma), south (Lubumbashi), west (Matadi), to the north (Mbandaka) and to the center (Mbuji-Mayi) but it is limited to three cities.

In conclusion, there is an urgent need to strengthen drug control capacity in the DRC both from a regulatory and technical point of view. An effective and adapted control will have consequences; improving the technical skills of local manufacturers, improving distributors' expertise in terms of drug preservation 
and discouraging counterfeiting of medicines in all its forms.

\section{Acknowledgements}

We thank the Belgium Academic research and higher education (Academique de Recherche et de l'Enseignement Supérieur or ARES-CCD) for supporting this work through fellow grants for N. Kalenda (SOFT program) and P. Ciza (PFS2016 project). Our gratitude is directed to the Health Ministry responsible in Kinshasa, Lubumbashi, Kolwezi and Matadi for their cooperation for sample collection and legal authorization.

\section{Conflicts of Interest}

The authors declare no conflicts of interest regarding the publication of this paper.

\section{References}

[1] Nayyar, G.M.L., Breman, J.G. and Herrington, J.E. (2015) The Global Pandemic of Falsified Medicines: Laboratory and Field Innovations and Policy Perspectives. The American Journal of Tropical Medicine and Hygiene, 92, 2-7. https://doi.org/10.4269/ajtmh.15-0221

[2] Taylor, R.B., et al. (2001) Pharmacopoeial Quality of Drugs Supplied by Nigerian Pharmacies. Lancet, 357, 1933-1936. https://doi.org/10.1016/S0140-6736(00)05065-0

[3] Johnston, A. and Holt, D.W. (2013) Substandard Drugs: A Potential Crisis for Public Health.

[4] Fadeyi, I., Lalani, M., Mailk, N., Van Wyk, A. and Kaur, H. (2015) Quality of the Antibiotics-Amoxicillin and Co-Trimoxazole from Ghana, Nigeria, and the United Kingdom. The American Journal of Tropical Medicine and Hygiene, 92, 87-94. https://doi.org/10.4269/ajtmh.14-0539

[5] Hajjou, M., et al. (2015) Monitoring the Quality of Medicines: Results from Africa, Asia, and South America. The American Journal of Tropical Medicine and Hygiene, 92.

[6] Pal, S., Dodoo, A. and Olsson, S. (2011) The World Medicines Situation 2011 Pharmacovigilance and Safety of Medicines.

[7] Ravinetto, R.M., Boelaert, M., Jacobs, J., Pouget, C. and Luyckx, C. (2012) PoorQuality Medical Products: Time to Address Substandards, Not Only Counterfeits. Tropical Medicine \& International Health, 17, 1412-1416. https://doi.org/10.1111/j.1365-3156.2012.03076.x

[8] Belew, S., et al. (2015) Assessment of Efficacy and Quality of Two Albendazole Brands Commonly Used against Soil-Transmitted Helminth Infections in School Children in Jimma Town, Ethiopia.

[9] Renschler, J.P., Walters, K.M., Newton, P.N. and Laxminarayan, R. (2015) Estimated Under-Five Deaths Associated with Poor-Quality Antimalarials in SubSaharan Africa. The American Journal of Tropical Medicine and Hygiene, 92, 119-126. https://doi.org/10.4269/ajtmh.14-0725

[10] Newton, P.N., et al. (2011) The Primacy of Public Health Considerations in Defining Poor Quality Medicines Poor Quality Medicines-A Major Public Health Prob- 
lem.

[11] Glass, B.D. (2014) Counterfeit Drugs and Medical Devices in Developing Countries.

[12] WHO (2010) WHO and Partners Accelerate Fight against Counterfeit Medicines. WHO.

[13] Delepierre, A., Gayot, A. and Carpentier, A. (2012) Update on Counterfeit Antibiotics Worldwide; Public Health Risks. Médecine et Maladies Infectieuses, 42, 247-255. https://doi.org/10.1016/j.medmal.2012.04.007

[14] Kelesidis, T., Kelesidis, I., Rafailidis, P.I. and Falagas, M.E. (2007) Counterfeit or Substandard Antimicrobial Drugs: A Review of the Scientific Evidence. Journal of Antimicrobial Chemotherapy, 60, 214-236. https://doi.org/10.1093/jac/dkm109

[15] You, D.Z., et al. (2015) Global, Regional, and National Levels and Trends in Under-5 Mortality between 1990 and 2015, with Scenario-Based Projections to 2030: A Systematic Analysis by the UN Inter-Agency Group for Child Mortality Estimation. The Lancet, 386, 2275-2286. https://doi.org/10.1016/S0140-6736(15)00120-8

[16] Newton, P.N., Green, M.D. and Fernández, F.M. (2010) Impact of Poor-Quality Medicines in the "Developing" World. Trends in Pharmacological Sciences, 31, 99-101. https://doi.org/10.1016/j.tips.2009.11.005

[17] Taylor, D. (2014) RFID in the Pharmaceutical Industry: Addressing Counterfeits with Technology. Journal of Medical Systems, 38, 141.

https://doi.org/10.1007/s10916-014-0141-y

[18] Taylor, P. (2013) Simple Screen Detects Fake and Substandard Antibiotics.

[19] Fadlallah, R., El-Jardali, F., Annan, F., Azzam, H. and Akl, E.A. (2016) Strategies and Systems-Level Interventions to Combat or Prevent Drug Counterfeiting: A Systematic Review of Evidence Beyond Effectiveness. Pharmaceutical Medicine, 30, 263-276. https://doi.org/10.1007/s40290-016-0156-4

[20] El-Jardali, F., et al. (2015) Interventions to Combat or Prevent Drug Counterfeiting: A Systematic Review. BMJ Open, 5, e006290.

https://doi.org/10.1136/bmjopen-2014-006290

[21] USP-NF Online Login. http://www.uspnf.com/uspnf/login

[22] Benner, A. (1999) Sample Size Tables for Clinical Studies. (2nd edn). David Machin, Michael J. Campbell, Peter M. Fayers and Alain P. Y. Pinol, Blackwell Science Ltd., Oxford, 1997. No. of pages: $\mathrm{x}+315$. Price: $£ 45$. ISBN 0-86542-870-0. Statistics in Medicine, 18, 494-495.

https://doi.org/10.1002/(SICI)1097-0258(19990228)18:4<494::AID-SIM56>3.0.C $\underline{\mathrm{O} ; 2-\mathrm{T}}$

[23] Hubert, P., et al. (2004) Harmonization of Strategies for the Validation of Quantitative Analytical Procedures: A SFSTP Proposal-Part I. Journal of Pharmaceutical and Biomedical Analysis, 36, 579-586.

[24] JCGM:200 (2008) Evaluation of Measurement Data-Guide to the Expression of Uncertainty in Measurement. https://ncc.nesdis.noaa.gov/documents/documentation/JCGM_100_2008_E.pdf

[25] Feinberg, M. (2007) Validation of Analytical Methods Based on Accuracy Profiles. Journal of Chromatography A, 1158, 174-183. https://doi.org/10.1016/j.chroma.2007.02.021

[26] Newton, P.N., et al. (2009) Guidelines and Guidance Guidelines for Field Surveys of the Quality of Medicines: A Proposal. PLoS Medicine, 6, e1000052. https://doi.org/10.1371/journal.pmed.1000052

[27] World Health Organization (2009) Annex 2 Stability Testing of Active Pharma- 
ceutical Ingredients and Finished Pharmaceutical Products. WHO Technical Report Series, No. 953.

[28] Bogaert, J. and Halleux, J.-M. (2015) Territoires périurbains: Développement, enjeux et perspectives dans les pays du Sud.

[29] (2010) Republique Democratique du Congo Ministere de la Sante Publique Secretariat General.

[30] Mortality of Children in Second Demographic and Health Survey, D.R. Congo, 2013-2014 (Chp. 8, p. 113).

https://reliefweb.int/sites/reliefweb.int/files/resources/FR300_0.pdf

[31] Alkema, L., Chao, F., You, D., Pedersen, J. and Sawyer, C.C. (2014) National, Regional, and Global Sex Ratios of Infant, Child, and Under-5 Mortality and Identification of Countries with Outlying Ratios: A Systematic Assessment. The Lancet Global Health, 2, e521-e530. https://doi.org/10.1016/S2214-109X(14)70280-3

[32] Almuzaini, T., Choonara, I. and Sammons, H. (2013) Substandard and Counterfeit Medicines: A Systematic Review of the Literature. BMJ Open, 3, e002923. https://doi.org/10.1136/bmjopen-2013-002923

[33] Agence d'Information d'Afrique Centrale (2017) Faux médicaments: Les Etats africains s'engagent à lutter contre le Fléau. adiac-congo.com: Toute l'actualité du Bassin du Congo.

http://www.adiac-congo.com/content/faux-medicaments-les-etats-africains-sengage nt-lutter-contre-le-fleau-60850

[34] Médicaments Contrefaits Guide pour l'élaboration de mesures visant à éliminer les médicaments contrefaits Département des Médicaments essentiels et Politique pharmaceutique Organisation mondiale de la Santé Genève, Suisse. 